\title{
Perspective
}

\section{Preparing for the threat of a pandemic: a Canadian student's perspective}

\author{
Derek CK Ng
}

The International Institute of Infection and Immunity, Shantou, China

\begin{abstract}
Emerging infectious diseases, such as a pandemic influenza outbreak, pose a threat to humans and economies worldwide. A novel course at Shantou University's Medical College in China has provided me and nine other Canadian students with a unique opportunity to learn with experts in infectious disease. We are taught in a small group setting and our lecture material is supplemented with hands-on experience in the laboratory. Being placed in China offers us a distinct perspective on culture and social factors and their role in disease transmission mechanisms. This training course has offered me and the other students a deeper understanding of infectious disease in general and is likely to be an experience that we will carry with us in our future careers. This program is a step in a positive direction towards promoting interest in this field of scientific study in Canada.
\end{abstract}

Key Words: education, influenza, pandemic, student perspective

J Infect Dev Ctries 2009; 3(7):575-578.

Received 6 April 2009 - Accepted 27 June 2009

Copyright $\odot 2009 \mathrm{Ng}$. This is an open-access article distributed under the Creative Commons Attribution License, which permits unrestricted use, distribution, and reproduction in any medium, provided the original work is properly cited.

The World Health Organization (WHO) recently warned, "...infectious diseases are emerging faster and spreading more rapidly than ever before" [1]. This caution has been accentuated by the recent H1N1 influenza pandemic in the summer of 2009. However, despite the exposure and attention H1N1 has garnered from the mass media, this WHO warning applies to many other diseases as well. The technological advances of the modern world, such as commercial flights, have contributed towards this unprecedented increase in disease transmission.

As an undergraduate student at McMaster University, I was once assigned a position paper in an introductory virology class. The objective was to take a stance on whether the Canadian government should invest research in viruses that cause disease only in Canadians. My professor explained that federal research funding is provided to scientists through the Canadian Institutes of Health Research (CIHR). After receiving funds from the federal government, the CIHR allocated it across fourteen institutes. One of these is the Institute of Infection and Immunity, which within itself contains several more grant panels including Virology and Viral Pathogenesis. My professor further elaborated that during each grant cycle, typically 60 to 70 grants are reviewed. Of those reviewed, about 20 percent are funded due to financial constraint. Approximately half of all the grants currently funded deal with HIV research and very few investigate emerging infectious disease. I had learned in class that the 1918 influenza pandemic resulted in 20 million to 40 million deaths. Considering the predictions of a looming H5N1 avian influenza pandemic, it seemed strange to me that this research was neglected. I was not surprised when I later learned that there is a shortage of Canadian scientists trained in the field of emerging infectious diseases.

The battle against emerging infectious diseases has a long history and deep roots. One successful method of combating diseases is vaccination. It has proved to be quite successful in its ability to confer immunity in an individual. A classic example of this is the eradication of smallpox, as declared by the WHO in 1979 [2]. Preparation against emerging infectious diseases will require not only scientific manpower, but cooperation on the global scale as well. This is marked by the need for countries, including Canada, to collaborate and communicate research findings. Further, there is also a need to extract research from outside of the laboratory, in the wild, where many of these diseases can evolve and emerge. 
Table 1. A brief and selected outline of the theoretical and practice

\section{Theoretical}

Current H1N1 Swine flu pathogenesis and knowledge

Presentation of H1N1 microarray data

H5N1 pathology in human patients

Evolution of the immune system and their responses to pathogens

An introduction to Traditional Chinese Medicine (TCM)
Practical

Viral inoculation into chicken eggs

Literature review and student-led presentations

HA-test, chicken egg harvesting, histology

Cell growth, virus purification, vaccine preparation, mouse infections

A visit to a local hospital and TCM pharmacy
The International Institute of Infection and Immunity (IIII), a program within Shantou University's Medical College, is currently addressing this issue. I am one of ten undergraduate students from Canada selected to embark on a unique training experience. In Dr. David Kelvin's novel program, we are immersed in an extensive summer training course in Shantou, China, focusing on host immune responses and emerging infectious diseases. We have been greatly appreciative of the selection process thus far, as we cooperate exceptionally and have formed a cohesive bond. Each of us bring a unique perspective to viral pathogenesis, as we each possess a diverse background spanning across immunology, virology, medicine and biomedical engineering.

The IIII is a joint collaboration between Shantou Medical College, Shantou University, The University Health Network from Toronto, Canada, and the University of Hong Kong. This institute occupies a unique perch in its research as it is in a prime location for investigating zoonosis - the transmission of viruses from animals to humans. Outside the classroom and laboratories, we will also participate in various field trips to further our learning. We are being taught by a world-renowned international faculty, who are equally as diverse as we are. The professors are experts in fields such as pandemic diseases, parasitology, bacteriology, and immunology. In addition to dynamic lectures, we also have an opportunity to experience emerging infectious disease research firsthand. In Dr. Kelvin's schematic, we are being trained in a two-pronged learning approach, characterized by small-group learning (Table 1). This method consists of theoretical classes in the morning and practical experiences in the afternoon. In this way, we are engaged directly with the faculty, as well as each other.
The theoretical sessions consist of a professor facilitating group discussion through a case study, problem or task (Figure 1). The opportunity for collaboration between the students truly shines here, as we are each able to draw from our different experiences and research strengths. For example, we were presented with real cDNA microarray lab data from the lungs of $\mathrm{H} 1 \mathrm{~N} 1$ infected ferrets. We were each told to select two upregulated genes of interest to research from the entire list. This was followed by a class discussion regarding the gene or its associated protein's characteristics and its possible relevance in swine flu pathogenesis. Questions are strongly encouraged and it has been a reciprocal process between the professors and the students so far. The small-group learning environment promotes freethinking and stimulates problem-solving abilities in a scientific context.

Figure 1. A group of students discussH5N1 pathogenesis with the STU Dean of Medicine, Dr. Jiang Gu.

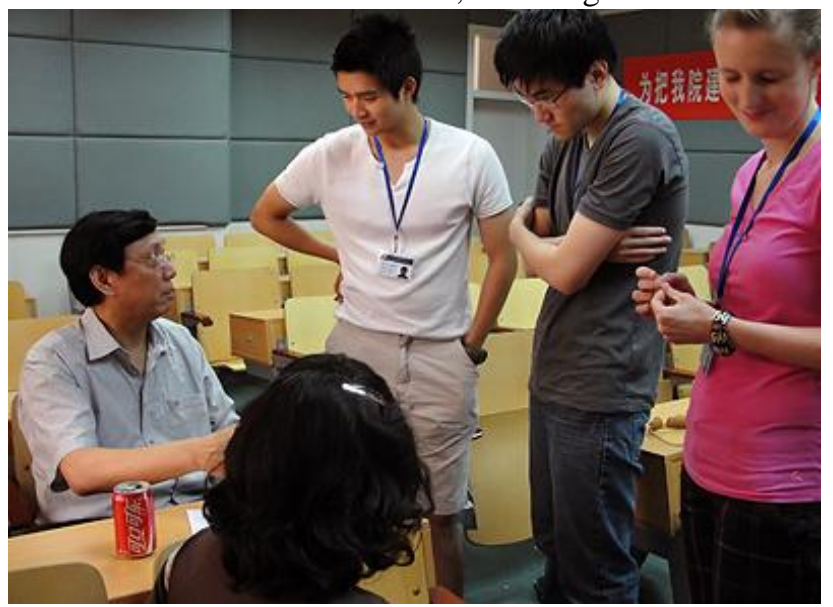

Practical experiences are invaluable to us as they solidify our theoretical knowledge. The students are broken into smaller groups and are mentored by postdoctoral members in the facility. Activities so far 
have included training in viral growth and vaccine production in cell culture and animal models (Figure 2). Ultimately, we will gain an appreciation for the various techniques used in studying viruses and diseases.

Figure 2. Students work closely with post-doctoral members in the lab, in this case growing influenza virus in chicken eggs.

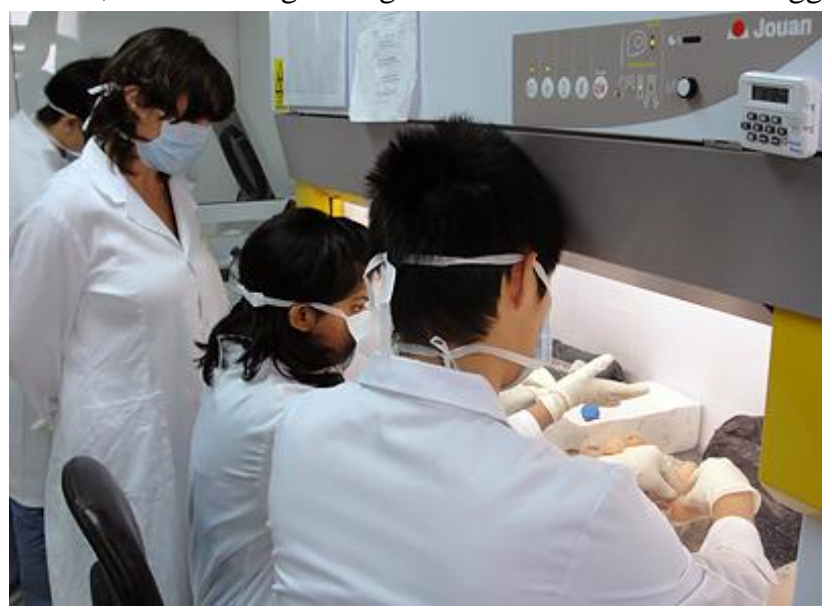

Since the program is administered in Shantou, China, we are very fortunate to be able to extend our learning outside the classroom and laboratory. Within the city, we are able observe textbook concepts in practice. For example, a trip to the marketplace can include sights such as various live-poultry species being housed together in close proximity to humans (Figure 3). We could then deduce the many ways in which the animals could transmit diseases to each other as well as to humans. Further, within one week of living in China, we have been able to appreciate the distinct differences in our cultures.

Figure 3. A common live poultry market consisting of duck, geese and chickens in Shantou, China.

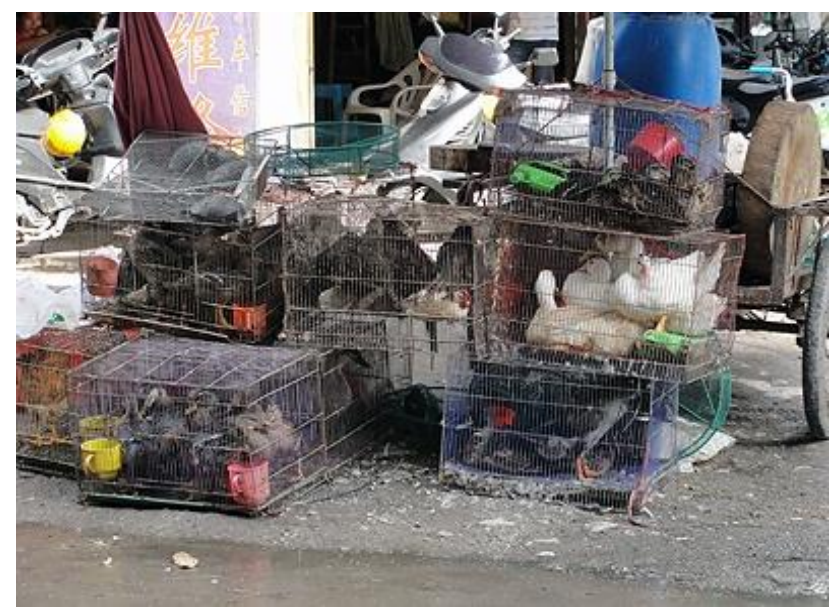

One unique aspect of living here in Shantou for one month is that we are not simply observing the culture - we are eating, drinking and breathing it each day. Living in China gives us the appreciation for the climate, insects and the level of sanitation here and how it ultimately relates to disease transmission. For example, during our Traditional Chinese Medicine module, I was able to visit a hospital in Shantou, China. Upon using the washrooms there, I was disturbed to find that the hospital did not provide hand soap. Despite the unorthodox toilets, lack of toiletries and the occasional traveler's diarrhea, I have been truly impressed by the students' determination to explore and sample everything that the city has to offer.

In addition to the aforementioned teaching schedule, Dr. Kelvin planned an extended field trip to Guilin, China. Just prior to the trip, Dr. Salvatore Rubino and Dr. Piero Cappucienelli instructed us on the topic of water-borne pathogens. This set the stage for the scenery that we would see next. Guilin was a fantastic city with lush green mountain peaks surrounding it. Prior to this experience, I had never seen such a beautiful sight in my life. We travelled along the Li River and while marveling at the sights, I thought about all of the cave openings in the mountains and how they likely led to labyrinths within. I further speculated towards the presence of bats and possible reservoirs for diseases. We also visited rice terraces, as suggested by Dr. Rubino. According to my GPS, we were $800 \mathrm{~m}$ above sea level at the peak. Along the paths up the mountain, I was able to notice the open sewage systems in place throughout the town. It was raining at the same time, and it was easy to see how the systems could flood and potentially contaminate food, local poultry or water supplies (Figure 4).

In the midst of a declared influenza pandemic, there is no better example of why there is a critical need for trained and prepared scientists. This course in Shantou is a step in the right direction towards promoting emerging infectious disease research in Canada. So far this excursion has truly been remarkable in provoking lateral thinking towards understanding the various factors that lead to disease transmission in populations. When we return home to our normal lives in August, I have no doubt that this course will be applicable in some way to our future 
Figure 4. Poultry eating rice from bamboo sticks over an open sewage system at a terrace in Guilin, China.

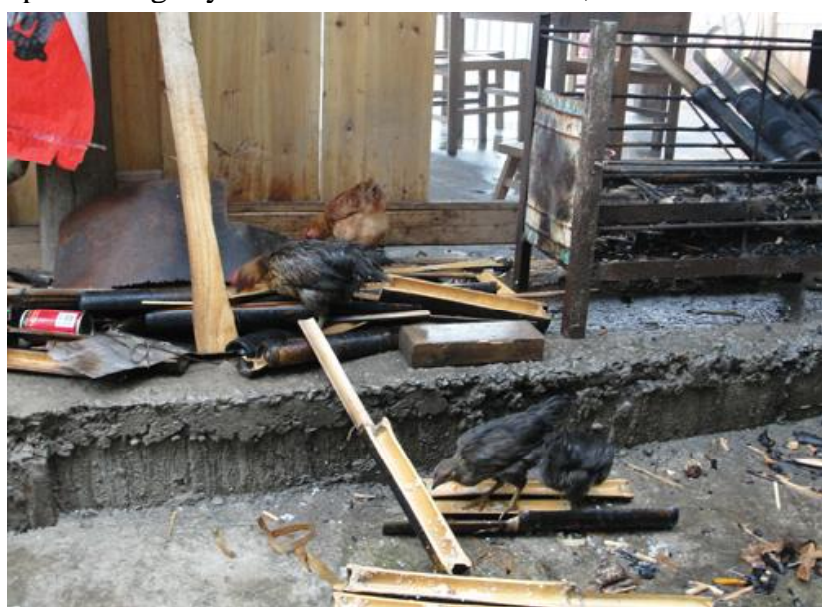

careers. John F. Kennedy once said, "The time to repair the roof is when the sun is shining." We must not hesitate to take action and prepare for the potential storm ahead of us.

\section{Acknowledgements}

Many thanks to my fellow colleagues from Canada: Fiona Almeida, Leigh Beamish, Timothy Chan, JoAnne Fernandes, Mackenzie Howatt, Joanne Leung, William Li, Theresa Robertson, Ben Wang. Thank you to Nikki Kelvin who offered guidance and revisions throughout this publication process. I would like to extend my gratitude towards the members and guest lecturers at the International Institute of Infection and Immunity for their wisdom and hospitality. Finally, my deepest gratitude goes to Dr. David Kelvin and our sponsors from the Li Ka Shing Foundation for providing us with this once-in-alifetime opportunity.

\section{References}

1. O'Dowd A (2007) Infectious diseases are spreading more rapidly than ever before, WHO warns. BMJ 335: 418.

2. Breman JG, and Arita I (1980) The confirmation and maintenance of smallpox eradication. N Engl J Med 303: 1263-73. 